Substituting from (4.1), we have

$$
\begin{aligned}
\chi^{(2)}=m(1- & \left.\left.m^{2}\right)^{-1 / 2} M y^{\prime-1 / 2} \pi^{-1} \int_{0}^{x^{\prime}} d \mu \int_{0}^{00 s^{-1}\left|y^{\prime} /\left(x^{\prime}-\mu\right)\right|} J_{0}\left[\kappa\left(x^{\prime}-\mu\right) \sin \theta\right)\right] \\
& \cdot \frac{\partial}{\partial \theta}\left\{\left[\left(x^{\prime}-\mu\right) \cos \theta-y^{\prime}\right]^{-1 / 2} w^{\prime}\left[\mu,\left(x^{\prime}-\mu\right) \cos \theta-y^{\prime}, \tau\right]\right\} d \theta
\end{aligned}
$$

The final solution for the pressure distribution on the upper surface of the wing, obtained by substituting $\phi^{(1)}$ in (2.8) and adding $\chi^{(2)}$ from (4.3), is given by

$$
\chi\left(x^{\prime}, y^{\prime}, 0+, \tau\right)=\left(1-m^{2}\right)^{-1 / 2} M\left[\phi_{x^{\prime}}^{(1)}\left\{w^{\prime}\right\}+m \phi^{(1)}\left\{w_{\nu^{\prime}}^{\prime}\right\}\right]+\phi_{\tau}^{(1)}\left\{w^{\prime}\right\}
$$

We remark that the results (4.3) and (4.5) are not restricted to harmonic time dependence, since they are valid for all frequencies.

In the case of a subsonic leading edge $(m \geq 0)$, it would be necessary only to replace $\phi^{(1)}\left\{w_{y^{\prime}}^{\prime}\right\}$ by $\phi_{y^{\prime}}^{(1)}\left\{w^{\prime}\right\}$ in (4.3).

\title{
REFERENCES
}

1. J. W. Miles, A general solution for the reclangular airfoil in supersonic flow, Q. Appl. Math., 11, 1-8 (1952).

2. P. A. Lagerstrom, Linearized supersonic theory of conical wings, J. P. L. Prog. Rep. 4-36 Pasadena, (1947); reprinted as NACA TN 1685 (1948), 90-98.

\section{NOTE ON THE MEAN SQUARE VALUE OF INTEGRALS IN THE STATISTICAL THEORY OF TURBULENCE*}

\author{
By C. C. LIN (Massachusetts Institute of Technology)
}

1. In the statistical theory of homogeneous isotropic turbulence, it is sometimes of interest to evaluate the mean square value of certain integrals, such as the pressure fluctuation over a sphere. The purpose of the present note is to give such an evaluation for integrals over a sphere and for similar integrals over spaces of other dimensions. The analysis shows that the final answer can be interpreted in terms of dimensional arguments; provided the length scale used is the geometrical mean of the scale of turbulence and the linear scale of the region over which the integral is taken. The results could be applied to the problem of the noise generated by turbulence.

2. Consider, for definiteness, the pressure fluctuation over the surface of a sphere. Extension to the study of other quantities can be easily made. Let the integral be denoted by

$$
I=\int p d S
$$

where $p$ is the pressure fluctuation at a point $P$, and the surface integral is extended over a sphere of radius $a$. We may also write

$$
I=\int p^{\prime} d S^{\prime}
$$

"Received April 20, 1953. 
where $p^{\prime}$ is the pressure fluctuation at a point $P^{\prime}$, and the integral is extended over the same surface of the sphere. Thus, if we calculate $I^{2}$ by multiplying (1) and (2), we obtain

$$
\left\langle I^{2}\right\rangle=\int d S \int\left\langle p p^{\prime}\right\rangle d S^{\prime}
$$

where \langle\rangle enclosing a quantity denotes a statistical average.

In isotropic turbulence, the statistical correlation $\left\langle p p^{\prime}\right\rangle$ depends only on the relative position of the two points $P$ and $P^{\prime}$. Thus, the integral

$$
J=\int\left\langle p p^{\prime}\right\rangle d S^{\prime}
$$

is independent of the position of the point $P$, and we have

$$
\left\langle I^{2}\right\rangle=4 \pi a^{2} J .
$$

To evaluate the integral $J$, we may take the point $P$ as the origin of a system of spherical coordinates. The element of area can be represented very simply if we consider zonal surfaces at a distance $r$ from the origin. In fact,

$$
d S=2 \pi r d r .
$$

Thus,

$$
J=2 \pi\left\langle p^{2}\right\rangle \int_{0}^{2 a} \odot(r) r d r
$$

where $\left\langle p^{2}\right\rangle$ is the mean square value of the pressure fluctuation, and $\mathcal{P}(r)$ is the correlation coefficient for pressure.

For very small spheres, we have the approximation

$$
\odot(r) \sim 1 \quad \text { for } \quad 0 \leq r \leq 2 a .
$$

Then $J$ may be approximated by

$$
J_{0}=4 \pi a^{2}\left\langle p^{2}\right\rangle
$$

and $\left\langle I^{2}\right\rangle$ may be approximated by

$$
\left\langle I^{2}\right\rangle_{0}=\left(4 \pi a^{2}\right)^{2}\left\langle p^{2}\right\rangle
$$

This also follows, if we write

$$
I=4 \pi a^{2} p
$$

for very small spheres.

For large spheres, $J$ may be approximated by

$$
J_{\infty}=2 \pi\left\langle p^{2}\right\rangle \int_{0}^{\infty} \rho(r) r d r .
$$

This may be written as

$$
J_{\infty}=4 \pi\left\langle p^{2}\right\rangle l^{2}
$$


where $l$ is a scale of turbulence defined by

$$
2 l^{2}=\int_{0}^{\infty} \odot(r) r d r
$$

Then

$$
\left\langle I^{2}\right\rangle=\left(4 \pi a^{2}\right)\left(4 \pi l^{2}\right)\left\langle p^{2}\right\rangle .
$$

The ratio $\left\langle I^{2}\right\rangle /\left\langle I^{2}\right\rangle_{0}$ is equal to

$$
\frac{\left\langle I^{2}\right\rangle}{\left\langle I^{2}\right\rangle_{0}}=\left(\frac{l}{a}\right)^{2}
$$

In the limit $a \rightarrow \infty$, this approaches zero. This is to be expected since $I / 4 \pi a^{2}$, being the average value of $I$ over the sphere, approaches zero when the sphere increases indefinitely in size.

In many cases, we are interested in averages over spheres of a size larger but comparable to the scale of turbulence; then (15) and (16) may be used as suitable approximations.

3. From the above arguments, it is obvious that the order of magnitude of $\left\langle I^{2}\right\rangle$ is independent of the detailed shape of the surface under consideration. Thus, we may write

$$
\left\langle I^{2}\right\rangle=k_{2}\left\langle p^{2}\right\rangle l^{2} L^{2},
$$

where $k_{2}$ is a constant, depending somewhat on the shape, $l$ is a scale of turbulence, and $L$ is a typical linear scale of the surface.

The formula (17) may be easily generalized to other dimensions. In general, we have

$$
\left\langle I_{n}^{2}\right\rangle=k_{n}\left\langle p^{2}\right\rangle(l L)^{n}
$$

for an integral

$$
I_{n}=\int p d \tau_{n}
$$

over an $n$-dimensional space. Thus, (18) can be obtained from (19) by dimensional arguments provided the volume integration is associated with the $n$th power of the length scale $(l L)^{\frac{1}{2}}$, i.e., the geometrical mean of the scale of turbulence and the linear scale of the region over which the integral is taken.

4. Some care must be exercised in estimating such integrals, if the divergence theorem can be used to convert them to integrals of lower dimensions. Consider, for instance, the integral

$$
K=\int \frac{\partial u}{\partial x} d \tau
$$

over a sphere. If we apply (18) directly to (20), we obtain

$$
\left\langle K^{2}\right\rangle=k_{3}\left\langle(\partial u / \partial x)^{2}\right\rangle(l L)^{3 / 2},
$$

or

$$
\left\langle K^{2}\right\rangle \sim \frac{\left\langle u^{2}\right\rangle}{\lambda^{2}} \cdot(l L)^{3}
$$


where $\lambda$ is Taylor's micro-scale of turbulence. However, this is actually incorrect. If we write (20) in the form

$$
K=\int u n_{x} d S
$$

then it becomes evident that

$$
\left\langle K^{2}\right\rangle \sim\left\langle u^{2}\right\rangle(l L)^{2} .
$$

The ratio between the two estimates $(21)$ and $(22)$ is $l L / \lambda^{2}$. It is clear that the direct application of the volume formula over-estimates the integral because of the small scale introduced by the differentiation process. Such occurrences are frequent in the statistical theory of turbulence. For example, the divergence of the Reynolds stress $\tau_{i i}=-\rho\left\langle u_{i} u_{i}\right\rangle$ is

$$
\frac{\partial \tau_{1 j}}{\partial x_{i}}=\frac{\partial}{\partial x_{i}}\left\{-\rho\left\langle u_{i} u_{i}\right\rangle\right\}=-\rho\left\langle u_{i} \frac{\partial u_{i}}{\partial x_{i}}\right\rangle .
$$

Based on the left-side, the estimate is

$$
\frac{\partial \tau_{i j}}{\partial x_{i}} \sim \frac{\tau}{l}
$$

while the right-side would give the (incorrect) estimate

$$
\frac{\partial \tau_{i i}}{\partial x_{i}} \sim \frac{\tau}{\lambda} .
$$

In all such cases, the lower estimates are to be taken.

\section{BOOK REVIEWS}

Gasdynamik. By K. Oswatitsch. Springer-Verlag, Wien, 1952. viii + 456 pp. $\$ 18.60$.

To write a comprehensive treatise on a rapidly expanding field of knowledge is not an attractive task: there is always the possibility that new developments may soon make the treatment appear dated or even incomplete. On the other hand, the lack of such a treatise may seriously impede the recruiting of new scientific talent, because newcomers will find it increasingly difficult to work their way through numerous important papers written in various languages and scattered over a great number of technical periodicals. In producing this comprehensive treatise on the dynamics of compressible fluids, the author has therefore rendered a significant service to all interested in the development of this branch of mechanics of continua.

Chapter I contains the necessary thermodynamic background. Chapters II and III are concerned with steady and unsteady flows in one dimension. The fundamental integral theorems are established in Chapter IV. These integral theorems remain valid in the presence of shocks; the differential equations of motion are readily derived from the integral theorems. Mechanical similarity is discussed, and various vortex theorems are presented. Chapter V illustrates the application of the integral theorems to technical problems. Chapter VI is devoted to the general equations for steady inviscid flow and to exact particular solutions of these equations (Prandtl-Meyer flow, axially symmetric conical flow, transformations of Molenbrock and Chaplygin, linearization of Prandtl and Glauert). Chapter VII is concerned with steady subsonic flows (plane or axially symmetric). In particular, the methods of Krahn, Janzen-Rayleigh, Kármán-Tsien, and Ringleb are discussed. Chapter VIII is devoted to steady supersonic flows in two dimensions (slightly disturbed parallel flow, shocks and their interaction, method of character- 